\title{
Enseñanza del emprendimiento en la educación superior: ¿Metodología o modelo?
}

\author{
María Eugenia Saldarriaga Salazar ${ }^{1}$ \\ Colegio Mayor del Cauca \\ msaldarriaga@unimayor.edu.co \\ María Fernanda Guzmán González² \\ Colegio Mayor del Cauca \\ mfdaguzman@gmail.com
}

DOI: https://doi.org/10.21158/01208160.n85.2018.2054

Fecha de recepción: 22 de diciembre de 2017

Fecha de aprobación: 07 de mayo de 2018

Cómo citar este artículo / To reference this article / Comment citer cet article / Para citar este artigo:

Saldarriaga Salazar, M.E. y Guzmán González, M. F. (2018). Enseñanza del emprendimiento en la educación superior: ¿Metodología o modelo? Revista EAN, 85, 125-142. DOI: https://doi.org/10.21158/01208160.n85.2018.2054

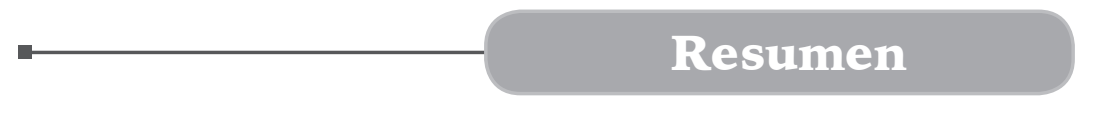

En el presente documento se presentan los resultados de un análisis de metodologías y modelos para la enseñanza del emprendimiento utilizadas en algunas universidades, entre los años 2008-2016. Para tal fin, se realizó una búsqueda de información sobre algunas universidades europeas, americanas, latinoamericanas, colombianas y del departamento del Cauca que incluyen el emprendimiento dentro de sus planes de estudio en diversas áreas. Luego de ello, se clasificó y analizó la información hallada y por último se identificaron las similitudes y diferencias existentes. Se encontró entonces que no son ni metodologías ni modelos lo que la mayoría de las universidades emplea para la orientación del emprendimiento, aunque algunas han hecho una recopilación de información para poder apropiar una metodología de enseñanza del emprendimiento.

\section{Palabras clave}

Emprendimiento - enseñanza, emprendimiento - metodología, emprendimiento - modelos de enseñanza, educación superior, universidades.

\footnotetext{
Magister en Dirección de Marketing, Universidad de Viña del Mar, Chile. Especialista Tecnológica en Administración de la Construcción, Institución Universitaria Colegio Mayor del Cauca, Colombia. Administradora de Empresas, Institución Universitaria Colegio Mayor del Cauca, Colombia. ORCID: http://orcid.org/0000-0002-1632-1058

2 Magister en Educación: Desarrollo Humano, Universidad de San Buenaventura. Licenciada en Lenguas Modernas: InglésFrancés, Universidad del Cauca. ORCID: http://orcid.org/0000-0001-6144-1465
} 


\title{
The teaching of entrepreneurship in higher education: a model or a methodology?
}

\begin{abstract}
This paper shows the outcomes obtained from a deep study of models and methodologies based on the teaching of entrepreneurship in some higher education institutions, from 2008 to 2016. For this, an information search about universities in Europe, North America, Latin America, Colombia and in Cauca region is made, having entrepreneurship as part of their curricula in common. After that, the collected information is classified and analyzed, identifying existing similarities and differences. As a result, it is found that neither methodologies nor models are used by most institutions to teach entrepreneurship, even though some of them collect information to adopt entrepreneurship teaching methodologies.
\end{abstract}

Key words. Entrepreneurship- teaching, entrepreneurship- methodology, entrepreneurship- teaching models, higher education, universities

\section{Apprendre l'entrepreneuriat dans l'enseignement supérieur: Méthodologie ou modèle?}

Résumé. Cet article présente les résultats d'une analyse des méthodologies et modèles d'enseignement de l'entrepreneuriat utilisés entre 2008 et 2016 dans certaines universités. À cette fin, plusieurs recherches d'informations ont été effectuées dans différentes universités européennes, américaines, latino-américaines et colombiennes qui mettent en avant l'esprit d'entreprise dans les cursus de différentes formations. Les informations obtenues ont ensuite été classifiées et analysées de manière à pouvoir en identifier, en extraire similitudes et différences. Le constat final de l'étude montre que l'orientation entrepreneuriale des cursus universitaires n'est ni le fait de la conséquence de la méthodologie utilisée ni celui des modèles que la plupart des universités mettent en place, même s'il a été posible de constater que certaines universités aient pu rassembler des informations leur permettant de s'approprier une méthodologie d'enseignement de l'entrepreneuriat.

Mots clefs. Entrepreneuriat - apprentissage; entreprenariat - méthodologie; entrepreneuriat - modèles d'enseignement; enseignement supérieur; universités.

\section{Ensino do empreendimento na educação superior: metodologia ou modelo?}

Resumo. No presente documento apresentam-se os resultados de uma análise de metodologias e modelos para o ensino do empreendimento utilizadas em algumas universidades, entre os anos 2008-2016. Para este fim, realizouse uma busca de informação sobre algumas universidades europeias, americanas, latino-americanas, colombianas e do departamento do Cauca que incluem o empreendimento dentro de seus planos de estudo em diversas áreas. Depois disso, classificou-se e analisou-se a informaç̧ão encontrada e por último identificaram-se as semelhanças e diferenças existentes. Encontrou-se então que não são nem metodologias nem modelos o que a maioria das universidades emprega para a orientação do empreendimento, ainda que algumas tenham feito uma recopilação de informação para poder apropriar uma metodologia de ensino do empreendimento.

Palavras-chave. Empreendimento - ensino, empreendimento - metodologia, empreendimento - modelos de ensino, educação superior, universidades. 


\section{Introducción}

$\mathbf{E}_{\text {emprendimiento en algunos países de }}^{n}$ Europa, América del Norte y Sur América se ha convertido en una prioridad; se ha establecido en algunos de estos como política pública, ya que lo que se requiere son personas con un cambio de mentalidad con la capacidad de formar empresas y generar empleos que contribuyan así a la dinamización de la economía, a la conexión con mercados internos y extranjeros y apoyar el desarrollo de las regiones. En Colombia, según el modelo GEM (2016) los emprendedores han generado emprendimientos económicos y sociales, dentro de sus propias empresas o como empleados, impulsando innovación, acelerando cambios económicos y sociales, promoviendo la competencia, la productividad e incrementando el desarrollo social y económico. Con respecto a la educación se determina que un gran número de nuevos emprendedores cuentan con formación universitaria tanto en pregrado como en posgrado.

La política pública pretende crear una cultura emprendedora, formar en aptitudes $\mathrm{y}$ actitudes personas con las capacidades y habilidades para ser transformadoras a través de la motivación, creatividad e innovación, que sean conocedoras del contexto en el que se desenvuelven y con una visión para establecer qué clase de emprendimientos serán los aptos para la región; se busca además establecer desde cuándo se da inicio a enseñar emprendimiento o se empieza a incluir en los planes de estudio y de qué manera se enseña en las diversas universidades identificadas.

Esta revisión de metodologías o modelos que se aplican en la enseñanza del emprendimiento surgen del proyecto de investigación «Diseño del modelo de enseñanza para el emprendimiento de la Institución Universitaria Colegio Mayor del Cauca», con el fin de generar cultura emprendedora y potenciar el desarrollo empresarial de los estudiantes. Por lo cual se hace una verificación de cómo enseñan el emprendimiento en las universidades, tanto internacionales como nacionales, se logra evidenciar que en su mayoría, emplean estrategias o guías para generar ideas de negocios, haciendo uso de los paso a paso como conocer el mercado, identificar clientes potenciales, generar ideas innovadoras, entre otros.

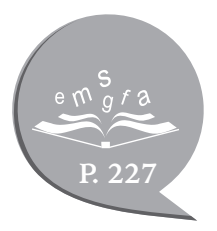




\section{Metodología}

L a investigación se sitúa en un enfoque del ámbito crítico-reflexivo sobre la forma cómo se enseña el emprendimiento en las diferentes universidades en el ámbito global, con esta información se busca indagar sobre qué, cómo, por qué y para qué se hace la generación de ideas o negocios. Las universidades se seleccionan teniendo en cuenta que estas tengan en sus currículos inserto el emprendimiento, y que sea en un periodo de 2008-2016, para lograr una visión más amplia se identifican universidades europeas, americanas, latinoamericanas, colombianas, y por último las del ámbito local en el departamento del Cauca, que es dónde se desarrolla la investigación macro que da pie a este documento.

La técnica de recolección de información se realiza con un proceso analítico, crítico y estructurado mediante una metodología de enfoque cualitativo, apoyada en información primaria de las universidades y secundaria como referencias bibliográficas. En este sentido, se complementan los datos que permiten comprender las prácticas y experiencias que son empleadas en las diversas universidades que posibilitan en los estudiantes la idea para desarrollar más emprendimientos con características creativas e innovadoras en el medio. La información se obtiene por medio de la búsqueda de datos o registros escritos que permiten establecer cómo se enseña el emprendimiento, se utiliza como referencia datos de diferentes autores que apoyan el tema a investigar, artículos relacionados con el tema, bibliografías, manuales, tesis de grado aplicadas en universidades de la ciudad, internet entre otros.

\section{Desarrollo del contenido}

$\mathbf{P}_{\mathrm{d}}$ artiendo del objetivo de la investigación, donde se pretende establecer si son modelos o metodologías la forma cómo se enseña el emprendimiento en diferentes universidades del ámbito global; a continuación se realiza un acercamiento teórico de los diferentes aspectos relacionados con la temática:

\subsection{Emprendimiento}

La palabra se define por primera vez bajo el concepto de «la persona que emprende y se determina a hacer y ejecutar, con resolución y empeño, alguna operación considerable y ardua» (Diccionario de la Lengua Castellana, 1732, p. 417).
También lo menciona (Cantillon, 1755) haciendo referencia a «la persona que paga un cierto precio para revender un producto a un precio incierto, por ende, tomando decisiones acerca de la obtención y el uso de recursos, y admitiendo consecuentemente el riesgo en el emprendimiento» (p. 21).

Say (1803) presenta al entrepreneur como el que "dirige una empresa, especialmente un contratista, actuando como intermediario entre el capital y el trabajo» (p.96), donde lo importante es que «el empresario está expuesto a todos los riesgos, por lo que se aprovecha de todo lo que puede serle favorable» (p. 153). Esa concepción perduró hasta comienzos del siglo $\mathrm{XX}$, y se puede resumir como la del propietario que maneja empresas y asume riesgos. 
Basados en estas definiciones, se puede inferir que el emprendedor es quien tiene una idea de negocio, la pone en marcha, asume los retos, hace una inversión de capital propio o préstamo-, con el fin de obtener una ganancia y, en algunos casos, generar empleos y se contribuya por medio de este a un mejoramiento de niveles sociales y el entorno económico de la región. Del mismo modo, es la persona que asume el riesgo, ya que además de la probabilidad que el negocio perdure y genere esas ganancias, está el otro lado, que el negocio no prospere y haya pérdidas o endeudamientos.

En ese sentido, se busca que los emprendedores sean personas creativas, innovadoras, con una alta motivación para emprender y no desfallecer en el primer momento. Así, para Schumpeter (1911), el emprendedor como agente de desequilibrio económico, es decir, los productos o servicios que el emprendedor genera contribuyen a desestabilizar la estructura del mercado ya que establece nuevas relaciones entre los diversos factores, creando nuevas iniciativas en un círculo virtuoso de nunca acabar, como lo mencionara Pitcher (2011) citado por Rodrigo (2011), esta relación innovaciónemprendimiento, es confirmada por Drucker (1985), quien manifiesta que el emprendedor no es aquel que desarrolla una empresa, sino el que aprovecha las oportunidades para generar espacios de innovación permanente inclusive más allá del ámbito económico (Formichella, 2004).

\subsection{Enseñanza de emprendimiento}

Los avances significativos de los emprendedores y sus emprendimientos generan la necesidad de brindar una formación con conocimientos más avanzados y con mayor profundidad, que les permiten ponerlos en práctica; además, ofertar programas que promuevan en la sociedad la generación de emprendimientos sostenibles, innovadores, creativos, entre otras características, esta promoción del conocimiento pretende favorecer sociedades con economías estables, ya que el conocimiento se constituye como una inversión muy productiva, estratégica en lo económico y prioritaria en lo social (Unesco, 2014); y por medio de la educación se logra apalancar el desarrollo de sociedad a partir de las oportunidades que tiene una persona para poder desarrollar y potencializar sus capacidades (Rodríguez, 2016).

Así, Campos y Méndez (2013) manifiestan que la enseñanza del emprendimiento debe ir direccionada hacia la formación de ciudadanos emprendedores desde una perspectiva autopoiésica del emprendimiento, asumiendo la enseñanza como un enfoque didáctico el aprendizaje basado en problemas (ABP). El proceso de formación de ciudadanos emprendedores requiere jóvenes que reconozcan sus derechos y deberes, resuelvan problemas y desarrollen creatividad sostenida como aspectos inherentes a su formación emprendedora, vinculada al desarrollo regional en términos económicos, sociales y ambientales. Flórez (1994) define la enseñabilidad como la base fundamental de la reflexión pedagógica contemporánea. Propone explicarla como una característica derivada del estatuto epistemológico de cada ciencia o disciplina, referida a los rasgos de racionalidad, sintaxis, de contenido teórico y experiencial que distingue el abordaje de problemas y condiciones, «específicamente la manera como cada disciplina puede o debe enseñarse» (p. 343).

Nuñez (2014) expone que en general los docentes que se dedican a la formación de emprendedores vienen de la formación académica, la investigación sobre cuestiones del emprendedorismo y la consultoría de empresas. No considera que la experiencia como empresario o haber emprendido la creación de una empresa por cuenta propia, se trate de una condición necesaria para poder «enseñar 
emprendedorismo». Aunque reconoce útil el contacto con experiencias emprendedoras, en su opinión, se trata de una perspectiva que va a depender del antecedente y experiencia profesional de cada profesor.

Cornella (2014) manifiesta que enseñar a emprender es el nuevo reto de los modelos educativos, debido a que la educación debe prepararse para formar en un mundo mucho más complejo; y el modelo más completo es aquel basado en problemas y proyectos. Según Foss y Klein (2004), citados por Orozco, Parra, Ruíz y Matíz (2016), el espíritu empresarial se ha convertido en una de las temáticas más desarrolladas en la economía moderna.

Megias (2013) argumenta que una educación emprendedora se basa en ayudar a los niños a desatar el potencial emprendedor que tienen dentro de sí, a enseñarles que no pasa nada si se equivocan, que el explorar y recorrer el camino es tan importante como llegar a la meta, el dinero es algo sustancial que deben entender, y que no todos los empresarios son unos aprovechados, sino que muchos son personas como ellos que decidieron dar un paso adelante haciendo realidad sus sueños, cuando ganan dinero se preocupan en generar empleo y valorar la sociedad, lo cual implica trabajar en varias direcciones habilidades, actitudes y conocimientos.

Baron y Shane (2008) manifiestan que la enseñanza emprendedora es un pilar en la educación; Neck y Green (2011) citados por Krauss (2011), reconocen que para formar emprendedores se necesita una metodología que depende de las personas, pero no del tipo de persona, y que para enseñar sobre emprendimiento se requiere práctica, así mismo, resalta que el rol de la universidad es formar emprendedores. Sin embargo, la manera de cómo se debe promover el espíritu desde la Universidad es aún un área escasamente tratada (Martín, Hernangómez y Rodríguez, 2005).

\subsection{Modelo de enseñanza}

Se considera un modelo de enseñanza como un plan estructurado que puede usarse para establecer un currículum, para diseñar materiales y orientar la enseñanza en las aulas. No existe un modelo generalizado para todos los tipos y estilos de aprendizaje, no se debe limitar a un modelo único, por llamativo que sea (Joyce y Weil, 1985). Enseñar desde una perspectiva general, es comunicar conocimientos, habilidades o actitudes. Los elementos fundamentales para un modelo son: enfoque, metodología y evaluación, estos elementos facilitan identificar qué modelo de enseñanza se está empleando, aunque en algunos casos se combinan elementos de cada modelo y se puede dar otro modelo aparentemente diferente.

Oser y Baeriswyl (2001), establecen que los modelos de enseñanza son una actividad generalizada donde los docentes de todos los niveles educativos toman sus procesos de enseñanza aprendizaje desde diferentes modelos. Estos modelos están de cierta forma relacionados y se fundamentan en teorías, permitiendo a los docentes ejercer su profesión. Martínez (2004) analiza la crítica de las instituciones educativas respecto a los contenidos frente a la vida cotidiana con la necesidad de construir relaciones en las aulas, teniendo en cuenta adecuación, intuición y reto que en el aula se equiparan a contextos, niveles, materias y alumnos, también indicadores de lo que es y significa la adaptabilidad de los modelos de enseñanza. 


\subsubsection{Características del modelo de enseñanza}

Acorde con lo establecido por Joyce y Weil (1985), se puede resumir las características del modelo de enseñanza de la siguiente manera:

- Conseguir objetivos de aprendizaje y desarrollo predeterminados.

- Orientación hacia la práctica.

- Diseño educativo para facilitar el conocimiento y desarrollo humano - fases del modelo.

- Diseño de ambientes de enseñanza.

- Herramientas de enseñanza.

- Promover el autoaprendizaje.

- Plantear hipótesis y teorías para dar posible solución a problemas del entorno social.

- Evaluación del proceso de enseñanza.

\subsection{Modelos de negocios adaptados para la enseñanza del emprendimiento}

Para Timmers (1998) un modelo de negocio es "una arquitectura de productos, servicios y flujos de información incluyendo una descripción de varios actores del negocio y sus roles, una descripción de los beneficios potenciales de diferentes actores del negocio y la descripción de las fuentes de ingreso» (p. 2). Es decir, la estructura del negocio que contempla la totalidad de los aspectos importantes a tener en cuenta para asegurar el éxito de este.
Andersson (2006) indica:

Los modelos de negocios se crean con el fin de dejar claro quiénes son los actores empresariales que se encuentran en un caso de negocio y cómo son sus relaciones explicitas. Las relaciones en un modelo de negocio se formulan en términos de valores intercambiados entre los actores. (p. 482)

En otras palabras, el modelo de negocios establece relaciones entre actores y sus funciones.

De los autores anteriores se pueden identificar los elementos de un modelo de negocios así:

- Propuesta de valor.

- Segmentación de mercados.

- Canales de distribución y comunicación.

- Relación con el cliente.

- Ingresos.

- Recursos claves.

- Actividades.

- Socios estratégicos.

- Estructura de costos.

En algunas universidades se han apropiado modelos de negocios, como por ejemplo el modelo Canvas, que han sido adaptados como modelos de enseñanza del emprendimiento, olvidando así la función fundamental de un modelo de enseñanza y preparando estudiantes para elaborar modelos de negocios sin enseñar las bases del emprendimiento. A continuación, se referencian algunas de las universidades analizadas y los modelos empleados para la enseñanza del emprendimiento.

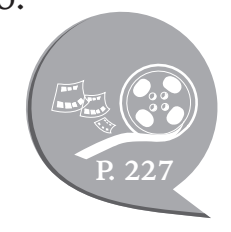




\subsection{Modelos de enseñanza del empren- dimiento en el ámbito internacional}

\subsubsection{Universidades europeas}

Universitat Politécnica de Catalunya. España. Favorece la viabilidad a los emprendedores desde un enfoque de negocio: modelo de negocio, tamaño del mercado -qué necesidades cubre, rasgos diferenciales, protección de la tecnología, etc.-, la comercialización, las finanzas, retroalimentación primeros usuarios/ clientes, ampliar desarrollo tecnológico, testeo del producto/beta.

Según el modelo anterior, se puede establecer que el diseño es un modelo de negocio adaptado a la enseñanza del emprendimiento, mas no un modelo de enseñanza en sí, debido a que se evidencian elementos del modelo de negocio como: propuesta de valor, segmentación del mercado, canales de distribución y comunicación, ingresos, relación con el cliente, estructura de costos.

\subsubsection{Universidades latinoamericanas}

Instituto Tecnológico y de Estudios Superiores de Monterrey, México. Con la intención de impulsar la formación para el desarrollo y el fortalecimiento del espíritu emprendedor, se tiene un modelo único de actividades curriculares transversales, desde las diferentes disciplinas del conocimiento, con sentido humano y visión global; retos y experiencias de emprendimiento, tales como: Bus Challenge, Hackatones, Hult Prize, Business Model Competition, Premio Santander a la Innovación Empresarial, Bootcamps; así como la participación en actividades emprendedoras con grupos estudiantiles, entre otros.

Modelo del proceso emprendedor. Alianzas para el Emprendimiento y la Innovación - AEI, Ecuador (2012): se enfoca en el momento de emprender, para ello se genera un proceso sistemático de cinco pasos: gestación, puesta en marcha, desarrollo inicial, crecimiento y consolidación, internacionalización. El desarrollo de competencias se realiza en la fase de gestación para el emprendedor, pero no describen ni identifican el tipo de competencias.

Universidad Católica de Uruguay. Emplean el modelo de negocio Canvas, que cuenta con 9 pasos: a) segmentar los clientes, para conocer el nicho de mercado y las oportunidades de nuestro negocio; b) definir bien la propuesta de valor, en otras palabras, saber por qué son innovadores y qué los diferencia de la competencia y los acerca a potenciales clientes; c) delimitar los canales de comunicación, distribución y de estrategia publicitaria a seguir, para fortalecer la marca e idea de negocio; d) establecer la relación que se mantiene con los clientes; e) determinar las fuentes económicas de la idea de negocio, un aspecto fundamental si se quiere tener éxito; f) identificar los activos y recursos clave que necesiten como piezas imprescindibles en el engranaje de la idea empresarial; g) conocer las actividades clave que darán valor a la marca, y saber las estrategias necesarias para potenciarlas; $h$ ) tener en cuenta los socios clave con los que establecer contactos y alianzas para el negocio. En otras palabras, definir las estrategias de networking con potenciales socios o proveedores, entre otras figuras importantes; i) marcar las estructuras de costos, para llegar a saber el precio que tendrá que pagar el cliente por adquirir el bien o servicio que ofrecerá la idea de negocio.

Universidad Nacional de General Sarmiento, Argentina. Promover el desarrollo emprendedor: programa de desarrollo emprendedor, basado en un modelo de trabajo que privilegia la articulación entre investigación, formación de recursos humanos y asistencia técnica a instituciones y emprendedores. Se da importancia estratégica a la participación 
en redes y alianzas con otras instituciones nacionales e internacionales, profesionales y emprendedoras, para lograr una mejor vinculación de ida y vuelta entre el proceso de construcción de conocimiento y la práctica concreta.

Dentro de las universidades latinoamericanas se logra establecer que el Instituto Tecnológico y de Estudios Superiores de Monterrey, México, y la Universidad Nacional de General Sarmiento, Argentina, sí conservan algunas características de un modelo de enseñanza de emprendimiento, tales como: obtener objetivos de aprendizaje y desarrollo predeterminados, orientación hacia la práctica, diseño educativo para facilitar el conocimiento y desarrollo humano, diseño de ambientes de enseñanza y herramientas de enseñanza.

Por otro lado, el modelo del proceso emprendedor Alianzas para el Emprendimiento y la Innovación - AEI, Ecuador y Universidad Católica de Uruguay reflejan la enseñanza del emprendimiento por medio de modelos de negocios, puesto que se tienen los elementos como: propuesta de valor, segmentación de mercados, canales de distribución y comunicación, relación con el cliente, ingresos, recursos claves, actividades, socios estratégicos y estructura de costo.

\subsubsection{Modelos nacionales}

Modelo Universidad EAN, Colombia. Desde sus inicios hasta la fecha ha pasado por diversos cambios hasta contar hoy en día con un modelo transversal aplicado a todos sus programas. Modelo de incubación en los últimos seis años; primero, con el programa Incuba-Innova-Integra (IN3), el programa Punto de Atención al Emprendedor (PAE) (Orozco et al., 2016), estos autores referencian que el modelo de incubación extramuros IN3, buscaba materializar las ideas emprendedoras en planes de negocio susceptibles a ser incubados y transformados en empresas de base tecnológica, a través de tres áreas de operación subdivididas en seis fases de incubación, que permitieron gestionar y viabilizar los planes de negocio de los emprendedores: El área de identificación y selección consistía en la inscripción y entrevista personal para la determinación de los requerimientos y necesidades de los planes de negocios inscritos. El área de incubación, por su parte, establecía las estrategias de asesoramiento y seguimiento para la creación de la empresa mediante la verificación del cumplimiento de un plan estratégico empresarial plasmado objetivamente en un plan de negocios. El propósito del área de gestión era apoyar a los participantes en la búsqueda de nuevos mercados y de fuentes de financiamiento, que apalancaran aquellos planes de negocios que estuvieran en una etapa de maduración avanzada para poder ser sostenibles a largo plazo. Este modelo se convirtió en la base de lo que hoy se conoce como EAN Impacta.

Modelo conceptual de desarrollo empresarial basado en competencias para la formación integral en ICESI. La universidad emplea el modelo de Timmons a la experimentación: contar con los recursos necesarios $\mathrm{y}$ un equipo que le ayude a conseguirlos y poner las propuestas en marcha, este modelo de establecimiento de empresas está basado en tres pilares: el mercado, las personas y los recursos; se considera el proceso de crear una empresa, hacerla crecer, capitalizar exitosamente, salir y comenzar de nuevo. Este modelo ha defendido en múltiples oportunidades que los emprendedores no nacen, sino que se forman, y que independientemente de la formación del individuo, en cualquier etapa de su vida puede adquirir la motivación para ser emprendedor. La universidad además emplea el aprendizaje activo, con el fin de realizar actividades de aprendizaje significativo, construir su propio aprendizaje, 
estimular a la investigación, responder a sus preguntas. Así mismo, el aprendizaje basado en proyectos para estimular y aprender el proceso del emprendedor, los estudiantes se involucran en la actividad experimental a través de proyectos para aplicar conocimientos previos en la solución de problemas.

Reinoso (2015) en su artículo se refiere al modelo de proceso empresarial propuesto por el Centro de desarrollo del Espíritu Empresarial de la Universidad ICESI (Colombia). Se presenta como un proceso de aprendizaje, con el propósito de desarrollar competencias emprendedoras y empresariales, compuesto por seis momentos pedagógicos autónomos: actitud emprendedora - etapa motivacional, el pensamiento creativo - etapa motivacional, el entorno de emprendimiento y empresarismo etapa situacional, el proceso de crear empresa - etapa sicológica, la idea de empresa - etapa analítica, el proyecto de empresa - etapa analítica y económica. Su objetivo de aprendizaje es motivar y orientar el emprendimiento de cada participante hacia la creación de empresa, mediante la oferta de unos espacios flexibles, motivadora y creativa de aprendizaje, orientadas car y desarrollar una actitud positiva de emprendimiento para identificar.

Guía de emprendimiento para crear tu propia empresa, Red Regional de Emprendimiento de Nariño, Colombia (2011) -para este artículo la guía se considera modelo porque contiene una estructuración curricular-. Plantean un instrumento para que los nariñenses desarrollen iniciativas de negocio, contribuyendo al fortalecimiento de los procesos empresariales que favorezcan a la región. Se establecen los siguientes pasos: sensibilización, ideas de negocio, plan de negocios, competencias del empresario y fuentes de financiación. Además, su propósito es acercar al individuo desde su formación teórica y práctica a la cultura de emprendimiento, generando el espíritu emprendedor, acondicionando los medios legales y financieros necesarios para su promoción y ejecución. Desarrollan las competencias emprendedoras después de realizar el plan de negocio.

Dentro de los modelos nacionales, se puede constatar que el modelo de la EAN conserva elementos de un modelo de negocio, tal vez por tener un modelo de incubación utilizado para la formación en emprendimiento. En ICESI y en la Red Regional de Emprendimiento de Nariño (2011), se revelan una combinación de modelos de negocio y modelos de enseñanza, al conservar la formación emprendedora con un diseño educativo, estableciendo los objetivos de aprendizaje con momentos pedagógicos autónomos para fortalecer el espíritu emprendedor, el desarrollo de las competencias emprendedoras, con el fin de lograr objetivos de aprendizaje y desarrollos predeterminados, posteriormente, se orientan los estudiantes a la práctica, para el desarrollo de una idea de negocio, y es aquí donde se aplican elementos de modelos de negocios con el fin de lograr que sus estudiantes creen empresas.

\subsubsection{Universidades departamento del Cauca}

Universidad del Cauca, Popayán. Garantizar emprendimientos empresariales y sociales. Enfoque conductual: asumir atributos y comportamientos para el rol de emprendedor.

Corporación Universitaria Autónoma del Cauca, Popayán. Está en estudio para proponer una modificación curricular que articule el emprendimiento como área de formación y el contexto económico y social del departamento del Cauca, así será posible establecer un acercamiento con la realidad del contexto, formar a los estudiantes para que asuman riesgos, sean emprendedores, establecer programas de capacitación en áreas acordes a los emprendimientos. 
En la Universidad del Cauca y la Corporación Universitaria Autónoma del Cauca, se deja entrever la aplicación de modelos de enseñanza de emprendimiento, porque constituyen diseños educativos al pretender modificar el currículo, esto conlleva al establecimiento de objetivos de aprendizaje, diseño de ambientes, herramientas y evaluación del proceso de enseñanza. Se busca fortalecer el rol emprendedor.

\subsection{Metodología de enseñanza}

Según Hernández (2001), una metodología didáctica supone una manera concreta de enseñar. Fidalgo (2007) expone que las metodologías educativas se fundamentan en las teorías del aprendizaje: conductismo, cognitivismo, constructivismo y el conectivismo. Sin embargo, Roing (2009) considera que la metodología como punto de fusión entre los objetivos y los temas para conceptualizar de una mejor manera los contenidos de aprendizaje al logro de los objetivos, representa la forma ideal para adaptar en materia de estudio las particularidades psicológicas del alumno, con el fin de alcanzar la eficacia en el proceso de enseñanza.

Las características de la metodología de enseñanza se establecen acorde a los autores mencionados:

- Enseñanza centrada en el estudiante.

- Labor formativa - docente.

- Contenidos.

- Uso de medios didácticos.

- Trabajo previo del estudiante.

- Mejorar capacidades de argumentativas reflexivas e interpretativas.

- Promover la investigación.

- Trabajo en equipo.

- Evaluación.
Las metodologías son una fuente de apoyo para mejorar los procesos de enseñanza como se ve a continuación:

\subsubsection{Metodologías internacionales}

\section{- Universidades latinoamericanas}

Universidad Metropolitana, Caracas, Venezuela. Aprendizaje basado en proyectos, temas relacionados con la realidad, propiciando la aplicación de conocimientos a propuestas novedosas. Estimular la capacidad de plantear y desarrollar soluciones adecuadas a problemas reales. Son base fundamental para el apoyo a los estudiantes en el desarrollo de sus ideas.

Universidad de Concepción de Chile. Metodología aprendizaje y servicio, es una técnica aplicada en que se desarrollan los principios del aprendizaje cooperativo, en todo momento y de forma transversal se trabaja el emprendimiento y trabajo en equipo interdisciplinario. Se aplican conceptos, procedimientos y actitudes de las 4 macro competencias genéricas, con especial énfasis en el pensamiento crítico, emprendimiento $\mathrm{y}$ trabajo en equipo interdisciplinario $\mathrm{y}$ responsabilidad social.

Universidad Católica de Uruguay. La metodología utilizada se denomina de aprendizajeservicio, es una propuesta pedagógica que favorece el crecimiento en valores y brinda la oportunidad a los estudiantes de colaborar con el desarrollo y mejora de la sociedad. Con el apoyo y seguimiento de sus profesores, viven su primera experiencia como consultores, brindando asistencia técnica en áreas afines administrativas, financieras, humanas y de marketing a microemprendimientos de escasos recursos. Favorece en los estudiantes la adquisición de una visión del mundo exterior ya que el mercado interno es muy pequeño 
Universidad de San Andrés, Argentina. Fomento al emprendimiento en la comunidad universitaria por medio de la cátedra Karel Steuer: identificar oportunidades, desarrollar nuevos proyectos, asumir riesgos, asumir compromisos éticos con la sociedad.

Universidad Federal de Santa Catarina. Universidad del Desarrollo del Estado de Santa Catarina. Universidad Estadual de Campinas. Universidad de Sao Paulo, Brasil. No hay metodología en sí; sin embargo, usan el estudio de casos, relatos de emprendedores, aspectos teóricos. Identificar oportunidades de negocio, nichos de mercado, organización, toma de decisiones y reflexión. Creación centro de incentivo a la asociación empresarial, con la finalidad de posicionarse como apoyo y fomento a la actividad empresarial en conjunto con el sector privado. Considera que el emprendimiento debe estar involucrado desde la infancia hasta en los programas de doctorado para expandir las misiones básicas de la universidad: enseñanza-investigaciónextensión. Competencias transversales por medio de proyectos conjuntos que involucren los programas, facultades y centros de la universidad.

En las universidades latinoamericanas identificadas, se demuestra que hay metodologías establecidas, como en la Universidad Metropolitana de Caracas, Venezuela, que utiliza el aprendizaje basado en proyectos que es una estrategia que se centra en el estudiante, ya que cada uno posee un cerebro único donde aprendizaje, habilidades y aptitudes tienen la misma importancia, los estudiantes tienen un papel activo y crítico para lograr estudiantes democráticos con pensamiento científico. La Universidad de Concepción de Chile y la Universidad Católica de Uruguay que aplican la metodología de aprendizaje y servicio, que es una propuesta educativa que articula aprendizaje y servicio a la comunidad, donde la formación debe conectar motivaciones y experiencias para ser significativa. Estas metodologías cumplen con las caracterizas identificadas anteriormente.

La Universidad Federal de Santa Catarina, Universidad del Desarrollo del Estado de Santa Catarina, Universidad Estadual de Campinas y la Universidad de Sao Paulo, Brasil, se enfocan en el estudiante, quien debe contar con una labor formativa desde la infancia, analizando estudios de casos que les permite mejorar capacidades argumentativas, reflexivas e interpretativas, por medio del trabajo en equipo llegan a la comunidad, cumpliendo así algunas de las características de la metodología de enseñanza.

Universidad de San Andrés, Argentina. Fomenta al emprendimiento en la comunidad universitaria, utiliza un modelo de negocios orientado por medio de una cátedra, al identificar oportunidades, desarrollar nuevos proyectos, asumir riesgos, asumir compromisos éticos con la sociedad, de esta manera asume elementos del modelo de negocios no de una metodología de enseñanza de emprendimiento.

\section{- Metodología nacional}

UniAmazonía, Florencia, Caquetá. En su PEI incorpora el desarrollo de «actitudes emprendedoras» como un elemento importante a tener en cuenta en la formación de sus estudiantes. Diseñaron su propuesta metodológica para la enseñanza del emprendimiento desde una dimensión didáctica -formación por competencias, enseñanza transversal durante la carrera; aprendizaje significativo y enseñanza para la comprensión-y una curricular -articulación de los cursos con énfasis en emprendimiento, incrementar proyectos de creación de empresa-.

UniAmazonía logra el diseño de su metodología combinando aprendizaje significativo y enseñanza para la comprensión 
desde la dimensión didáctica. Se centra en la formación de sus estudiantes desde las actitudes emprendedoras, pero al desarrollar el diseño curricular se introduce dentro de un modelo de enseñanza, donde se toman aspectos de un modelo de negocio promover la creación de empresas.

\section{Resultados}

$\mathbf{R}^{\mathrm{s}}$ especto al análisis realizado, se obtiene que se utiliza en la enseñanza del emprendimiento modelos y metodologías, pero estos son asociados a los modelos de negocios olvidando los aspectos fundamentales de la formación en emprendimiento que deben ser incluidos desde los aspectos curriculares.

\subsection{Hallazgos en el ámbito internacional}

En el ámbito internacional se observa cómo en países tanto de Europa como Norte, Centro y Sur América, evidencian la enseñanza del emprendimiento en su sistema educativo. Emplean el modelo de negocio como el Canvas y no un modelo de enseñanza de emprendimiento, haciendo una apropiación de este en el currículo de las universidades. De esta manera se olvida el fundamento teórico de un modelo de enseñanza. Así, con el fin de promover y poner en práctica estos procesos para la creación y puesta en marcha de los diversos emprendimientos, se fomenta la participación en concursos de ideas y planes de negocio para crear proyectos en forma conjunta, que sirvan de apoyo y complemento para su proceso final y perdurabilidad. De este modo, se permiten establecer negocios con nuevos socios para poder agregar valor real a las ideas de negocio existentes o venideras, las proyecciones que pretenden a futuro son: brindar apoyo financiero, establecer red de contactos y proveedores, ayudar con patentes y licencias, dejando de lado la formación de la persona emprendedora.
Otra metodología que se emplea es denominada de aprendizaje-servicio, usando el aprendizaje significativo para motivar a los estudiantes a aprender por competencias articulando la teoría y la práctica por medio de procesos de reflexión, es una propuesta pedagógica que favorece el crecimiento en valores, y brinda la oportunidad a los estudiantes de colaborar con el desarrollo y mejora de la sociedad. Con el apoyo y seguimiento de los profesores, los estudiantes viven su primera experiencia como consultores, brindando asistencia técnica en áreas afines administrativas, financieras, humanas y de marketing a microemprendimientos de escasos recursos. Impulsa además una conexión con el mundo exterior, otros mercados, ya que el interno es muy pequeño en algunos países, lo que les permite ampliar su visión.

Ciertos países presentan como metodologías el estudio de casos, relatos de emprendedores, aspectos teóricos que permiten identificar oportunidades de negocio, nichos de mercado, organización, toma de decisiones y reflexión. Esto favorece la formación de lazos con el sector productivo, y así se posibilita la capacitación a los empresarios tanto dentro de la comunidad educativa como el resto de la sociedad que tienen fines emprendedores.

En algunos países, las universidades han creado asociaciones para promover la acción empresarial con la finalidad de posicionarse como apoyo y fomento a la actividad empresarial en conjunto con el sector privado, ya que los 
consideran como impulso del autoempleo, así la gente además de crear empresa, genera empleos y contribuye al mejoramiento financiero de la región. Así, se pueden crear planes estratégicos de empresa según las necesidades que se observan en el entorno, donde se pueda aplicar los conocimientos formales en la búsqueda de soluciones concretas, temas relacionados con la realidad, propiciando la aplicación de conocimientos en propuestas novedosas aprendizaje basado en proyectos-.

Se considera que el emprendimiento debe estar involucrado desde la infancia hasta en los programas de doctorado para expandir las misiones básicas de la universidad: enseñanza-investigación-extensión; se debe contar con competencias transversales por medio de proyectos conjuntos que involucren los programas, facultades y centros de la universidad.

Fomento al emprendimiento en la comunidad universitaria por medio de la cátedra Karel Steuer: identificar oportunidades, desarrollar nuevos proyectos, asumir riesgos, asumir compromisos éticos con la sociedad.

Por otro lado, algunas instituciones no evidencian autores ni modelos a seguir, ya que aún no han dimensionado la necesidad de la formación de emprendedores como gestores y formadores de empresas para fortalecer el desarrollo económico de la región. Se ha ido incorporando en carreras como enfermería, periodismo y así crear realidades en el mercado.

\subsubsection{Convergencia}

Se usan metodologías como Canvas y ABP que permiten la apropiación de las competencias emprendedoras en la comunidad estudiantil, se cuenta con el apoyo de los profesores para que se fortalezca el desarrollo de ideas, se pongan en marcha, y los mismos estudiantes puedan servir de apoyo al sector empresarial público y privado del entorno. Se han creado grupos de apoyo dentro de las instituciones con el fin de promover la participación y puesta en parcha de los emprendimientos o ideas. La mayoría de universidades contribuye a estimular la competencia emprendedora en la comunidad estudiantil. Promoviendo y permitiendo a los estudiantes compartir sus ideas, se pretende generarlas, llevarlas a la realidad y asesorar o ayudar a conseguir recursos para poner en marcha los emprendimientos.

Sin embargo, a pesar que desde los años 90 se empieza a hablar del emprendimiento, aún no se le ha dado la importancia que tiene como medio de fortalecimiento para la economía de la región.

\subsection{Hallazgos en el ámbito nacional}

En Colombia se cuenta como política pública y ha sido incentivada por los avances internacionales.

Se entiende el emprendimiento o la consolidación de la formación empresarial como medio para potenciar el desarrollo económico de la región, por medio de la formación y desarrollo de competencias que permitan plantear alternativas factibles de solución a problemáticas propias de la realidad del estudiante, se promueve el trabajo en equipo.

Las instituciones trabajan con diversas metodologías, como el modelo Timmos: contar con los recursos necesarios y un equipo que le ayude a conseguirlos y poner las propuestas en marcha; $\mathrm{ABP}$ basados en la realidad, donde se busca dar soluciones a problemas del entorno; el aprendizaje activo: realizar actividades de aprendizaje significativo, construir su propio aprendizaje, estimular a la investigación, responder a sus preguntas. Modelo pedagógico basado en el desarrollo 
de competencias y enfocado a la formación integral y del espíritu empresarial; enfoque conductual: asumir atributos y comportamientos para el rol de emprendedor

Por otro lado, algunas instituciones han diseñado su propuesta metodológica para la enseñanza del emprendimiento desde una dimensión didáctica -formación por competencias, enseñanza transversal durante la carrera; aprendizaje significativo y enseñanza para la comprensión- y una curricular articulación de los cursos con énfasis en emprendimiento-, incrementar proyectos de creación de empresa, así favorece la creación de emprendimientos empresariales y sociales; el modelo EANTEC -modelo de formación en competencias emprendedoras de la universidad EAN-centrado en el desarrollo de competencias emprendedoras: proyecto de vida emprendedor, análisis del entorno para la identificación de oportunidades de negocio y el desarrollo de herramientas gerenciales para la planeación y puesta en marcha de empresas. La Universidad del Cauca está realizando un estudio para proponer una modificación curricular que articule el emprendimiento como área de formación y el contexto económico y social del departamento del Cauca.
El recurso de contar con diversas metodologías lleva al estudiante a que identifique nuevas y mejores oportunidades de negocio, analice la viabilidad y factibilidad de la futura empresa por medio de escenarios financieros y proyecciones, se ponga en marcha la idea de negocio, se fomente la búsqueda de apoyo financiero por medio de los laboratorios o la participación en eventos para que las empresas de los sectores público o privado tomen la iniciativa de invertir o apoyar.

Sin embargo, al haber aún deficiencia en la implementación de la ley, la creación de empresas es aún escasa y falta apoyo.

\subsubsection{Convergencia}

Se ha generado una cultura del emprendimiento, en donde las instituciones promueven y generan la creación de empresa, con ideas innovadoras y creativas que puedan competir en mercados nacionales e internacionales, así se logra impulsar el desarrollo de las regiones.

Sin embargo, a pesar de ser considerada como política pública, todavía no ha generado el impacto esperado, por ellos se han realizado seguimiento e instauración de la enseñanza del emprendimiento.

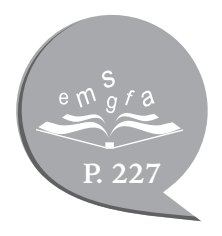




\section{Conclusiones}

$\mathrm{E}^{\mathrm{n}}$ n la mayoría de universidades se tienen en cuenta guías o estrategias para la formación de emprendedores; sin embargo, unas cuantas han hecho estudios de diversos métodos, metodologías y guías para crear sus propios modelos de enseñanza en emprendimiento y están siendo aplicados en las diversas carreras. Para la enseñanza del emprendimiento se han implementado modelos de enseñanza, metodologías de enseñanza y modelos de negocios, modelo de enseñanza porque se ha implantado desde el diseño curricular, se plantea el logro de objetivos, hay una orientación a la práctica en la generación de ideas de negocio, existen ambientes de aprendizaje y se evalúa el modelo establecido.

La metodologías se han caracterizado por pensar en la formación de los estudiantes como emprendedores, hay una labor formativa en los docentes, se establecen contenidos, hay un trabajo previo del estudiante, se mejoran capacidades argumentativas, reflexivas e interpretativas, y se evalúa la metodología. Todo esto ha generado la aplicación de modelos de negocios, puesto que se quiere llevar al estudiante a un proceso final cuyo resultado es la creación de empresa. Los modelos o metodologías que las universidades han apropiado, deben formar al emprendedor de una manera consiente, donde se les lleve a conocer su entorno, conozcan los problemas reales del mundo empresarial y puedan tomar decisiones acertadas, de tal manera que no desistan de continuar con sus emprendimientos y sigan buscando más oportunidades. Con lo anterior es importante precisar que los modelos de enseñanza contienen metodologías y esta a su vez los métodos o estrategias para lograr la enseñanza, en este caso de emprendimiento.

El auge del emprendimiento y su instauración como política pública en algunos países, ha generado que algunas universidades incluyan en sus programas de formación esta temática, ya sea para sus programas de administración o en forma transversal en todos sus programas, a fin de promover en sus estudiantes y futuros egresados el ser emprendedores en formar sus propias empresas. Por dar cumplimiento a la política se han tomado modelos de negocios, no se han construido modelos o metodologías de enseñanza del emprendimiento.

La mayoría de universidades han generado programas de apoyo a los estudiantes, con el fin de conseguir recursos para poner en marcha sus ideas de negocio, ser conocidas en el medio y tener proyección local, regional, nacional e internacional. Se le da más importancia a la creación del negocio en sí que a la formación del emprendedor, por lo que la sostenibilidad de los negocios se torna muy difícil.

Es importante reflexionar si todas estas apropiaciones que las universidades han realizado, para de una u otra forma enseñar el emprendimiento, qué tan efectivas han resultado, si logran la generación de una cultura emprendedora, y si los emprendimientos son sólidos y han logrado un posicionamiento en el mercado; o por el contrario el emprendimiento se enseña para cumplir un requisito más de sus currículos. 


\section{Referencias}

Alianzas para el Emprendimiento e Innovación. (2013). Ecuador, un país emprendedor e innovador en el 2020. Modelo del proceso emprendedor. Recuperado de http://aei.ec/wp/wp-content/uploads/2015/07/ ESTRATEGIA_EEI20201.pdf

Andersson, B., Bergholtz, M., Edirisuriya, A., Ilayperuma, T., Johannesson, P., Gordijn, J., Grégoire, B., Schmitt, M., Dubois, E., Abels, S., Hahn, A., Wangler, B., Weigand, H. (2006). Towards a Reference Ontology for Business Models. In D. W. Embley, A., Olivé, S. Ram (Eds.). Conceptual Modeling - ER 2006. ER 2006. Lecture Notes in Computer Science, 4215. Springer, Berlin, Heidelberg.

Baron, A., y Shane, S. (2008). Entrepreneurship, a process perspective. (2nd ed.). London: South-Western; [Thomson Learning distributor].

Cantillon, R. (1755). Ensayo sobre la naturaleza del comercio en general. México: Fondo de Cultura Económica.

Campos, O. R., y Méndez, G. C. (2013). La enseñanza del emprendimiento a partir del aprendizaje basado en problemas $(\mathrm{ABP})$ en la educación media técnica. Revista Amazonia Investiga, 2(2), Recuperado de http://www.udla.edu.co/revistas/index.php/ amazonia-investiga/article/view/15

Cornella, A. (2014). Enseñar a emprender. Los retos de los nuevos modelos educativos. EduCaixa. [Entrada de blog]. Recuperado de https://blog.educaixa.com/ es/-/jornada-ensenar-a-emprender-alfons-cornella

Diccionario de la Lengua Castellana. (1732). Tomo tercero. Madrid: Imprenta de la Real Academia Española.

Drucker, P. (1985). La innovación y el empresario innovador. Editorial Edhasa.

Fidalgo, A. (2007). Innovación educativa en la universidad. La asignatura pendiente. Bloque 1. Universidad Politécnica de Madrid. 49-54. Recuperado de https:// www.madrimasd.org/informacionidi/revistas/ monograficos/monografias/monografia20/20_ bloque1_07.pdf

Flórez, R. (1994). Hacia una pedagogía del conocimiento. Bogotá: McGraw-Hill.
Formichella, M. M. (2004). El concepto de emprendimiento y su relación con la educación, el empleo y el desarrollo local. Monografía. Buenos Aires Argentina. pp. 1-34.

Global Entrepreneurship Monitor - GEM. (2016). Reporte GEM Colombia 2015/2016.

Hernández, C. (2001). Metodologías de enseñanza y aprendizaje de altas capacidades. Ponencia. Facultad de Psicología, Universidad de La Laguna. España. Recuperado de https://gtisd.webs.ull.es/ metodologias.pdf

Joyce, B., y Weil, M. (1985). Modelos de enseñanza. New Jersey, Usa. Pretince Hall, Inc. Traducción de Ricardo Sánchez. Editorial Anaya.

Krauss, C. (2011). Actitudes emprendedoras de los estudiantes universitarios: el caso de la Universidad Católica del Uruguay. Revista Científica Dimensión Empresarial, 9(1), 28-40.

Martínez, V. N. (2004). Los modelos de enseñanza y la práctica de aula. Universidad de Murcia. Recuperado de http://www.um.es/docencia/ nicolas/menu/publicaciones/propias/docs/ enciclopediadidacticarev/modelos.pdf

Martín, N.; Hernángómez, J. J, y Rodríguez, A. I. (2005). Análisis de la formación y la experiencia laboral como determinantes del espíritu emprendedor de los estudiantes universitarios. Revista Asturiana de Economía-RAE, [Número especial], 34, 131- 145.

Megias, J. (2013). La educación para emprender, pilar de nuevos modelos de negocio. [Entrada de blog] Recuperado de https://javiermegias.com/blog/

Unesco. (2014). Educación para el Desarrollo Sostenible (EDS). Colombia: Unesco.

Orozco, J. A.; Parra, L. D.; Ruíz, C. L. y Matíz, F. J. (2016). Acompañamiento a emprendedores en la Universidad EAN: Del modelo IN3 a EAN Impacta 2011-2016. Revista Escuela de Administración de Negocios, (81), 12-25. Bogotá. http://dx.doi. org/10.21158/01208160.n81.2016.1553

Oser, F. K., y Baeriswyl, F. J. (2001). Choreographies of Teaching: Bridging Instruction to Learning. En Richardson V. (Dir). Handbook of Research on Teaching. American Educational Research Association, Washington, DC, p. 1031-1065. 
Pitcher, J. (2011). Innovación y destrucción creativa: la visión Schumpeteriana de espíritu empresarial. En R. Valera (Comp.), Desarrollo, Innovación y Cultura Empresarial (pp. 159-167). Cali: ICESI.

Red Regional de Emprendimiento de Nariño. (2011). Guía de emprendimiento para crear tu propia empresa. Recuperado de https://issuu.com/jdiazunimar/ docs/guia_emprendimiento/3

Reinoso, J. F. y Serna, L. F. (2015). Desarrollo de Competencias Emprendedoras a través de la Implementación de la Metodología de Aprendizaje denominada "de la Oportunidad al Emprendimiento". Revista de Economía \& Administración, 12 (1). 113-124

Rodríguez, A. M. (2016). Importancia del emprendimiento en la educación media en Colombia. (Trabajo de grado). Universidad Militar Nueva Granada. Recuperado de http://repository.unimilitar.edu.co/bitstream/10654/14203/3/Rodr\%C3\%ADguezChavesAnaMar\%C3\%ADa2016.pdf

Say, J. B. (1803). Traité d'économie politique. Osnabruck

Timmers, P. (1998). Business models for electronic markets. Electronic Markets, 8(2), 3-8. 